\title{
Innovación abierta en empresas colombianas: reto a superar*
}

\author{
Bernal-Torres, César Augusto* \\ Frost-González Salomón ${ }^{* \star *}$
}

\section{Resumen}

La importancia que en la actualidad otorgan las empresas de países desarrollados a la denominada innovación abierta es fundamental para fortalecer su capacidad innovadora. En tal sentido, este artículo analiza el uso que las empresas de países como Colombia hacen de las diferentes fuentes de innovación abierta para desarrollar sus innovaciones y el efecto que esas fuentes tienen en la rentabilidad y la participación de sus mercados. Para ello se aplicó una encuesta de percepción a 434 directivos de 53 empresas medianas y grandes, consideradas innovadoras, de diferentes sectores económicos de Bogotá, Colombia. El procesamiento de los datos se realizó mediante estadística descriptiva y el modelo de regresión lineal múltiple. Los resultados indican que las empresas estudiadas utilizan fuentes internas para sus innovaciones pero muy poco fuentes externas. Escasamente practican la innovación abierta, dado que continúan acentuando su labor en la innovación tradicional fundamentada en la generación, diseño y desarrollo de ideas surgidas en el interior de sí mismas. Esto de acuerdo con estudios recientes sobre innovación, es considerado un enfoque insuficiente para responder a los retos del actual ambiente de los negocios.

Palabras clave: Innovación abierta; fuentes de innovación; innovación tradicional; ambiente de los negocios.

Recibido: 25.09.14. Aceptado: 31.05 .15

* Este artículo es el resultado del desarrollo del proyecto titulado "IMPORTANCIA DE LA INNOVACIÓN ABIERTA EN LAS EMPRESAS EN PAÍSES EMERGENTES” realizado en alianza y con recursos de la Eicea y el Instituto de Postgrados - Forum de la Universidad de La Sabana- Colombia.

** Doctor en Administración de Negocios, Magíster en Educación, Economista y Psicólogo. Profesor Asociado de la Escuela Internacional de Ciencias Económicas y Administrativas (Eicea) de la Universidad de La Sabana, Chía, Cundinamarca, Colombia. Director del grupo de investigación Innovación y estrategia de esta Universidad. E-mail: cesar.bernal@unisabana. edu.co

*** Magíster en Administración de Empresas. Estudiante de doctorado en Educación, Universidad de La Salle. Decano de Posgrados de Administración de Negocios de la Universidad Sergio Arboleda, Bogotá, Colombia. E-mail: salomonfrost1@gmail.com. 


\title{
Open innovation in Colombian enterprises: Challenge to overcome.
}

\begin{abstract}
The importance currently given by developed countries enterprises to the named open innovation is fundamental to strengthen their innovative capacity. In that sense, this article analyzes the use that companies from countries such as Colombia make of the different open innovation sources in order to develop their innovations and the effect those sources have in the profitability and their markets participation. Therefore, a perception survey of 434 managers from 53 small, medium and large enterprises considered innovators from different economic sectors of Bogotá, Colombia was made. The data processing was carried out by descriptive statistic and the multiple linear regression model. The results indicate that the studied companies utilize internal sources for their innovations but very little external sources. They barely practice open innovation given that they continue to highlight their work on traditional innovation based on generation, design and development of ideas that have emerged from the inside of themselves. According to recent studies on innovation, this is considered an insufficient approach in the direction of responding to the current business environment challenges.
\end{abstract}

Key Words: Open innovation; innovation sources; traditional innovation; business environment.

\section{Introducción}

Nadie cuestiona, en la actualidad, la importancia de la innovación como variable estratégica para la competitividad empresarial y como el factor clave para el progreso de los países, pero sí la insuficiencia del modelo de innovación lineal tradicional soportado en los departamentos de investigación y desarrollo (I+D) de las propias organizaciones como la principal fuente de innovación.

Este modelo de $\mathrm{I}+\mathrm{D}$, exitoso durante gran parte del siglo $\mathrm{XX}$, está siendo cada vez más cuestionado debido a la demanda de grandes inversiones (para la disponibilidad de personal experto y para la dotación de laboratorios), la incertidumbre y los prolongados tiempos para la obtención de resultados que, en muchos casos, no garantizan el éxito en la innovación.

Para responder a las exigencias del nuevo ambiente de las organizaciones, en el campo de la innovación, ha surgido el enfoque de la innovación abierta que hace explícita la necesidad de apertura por parte de las empresas en sus procesos de innovación y pone énfasis en procesos como la práctica de rutinas de búsqueda de conocimiento externo como una de las principales fuentes para contribuir a potenciar la capacidad competitiva no sólo de las propias empresas, sino también, las de regiones y de economías nacionales.

Asimismo, este enfoque de la innovación abierta hace hincapié en la abundancia de conocimiento externo que las empresas pueden obtener para incrementar su capacidad innovadora en cualquiera de sus distintas actividades -producto, proceso, marketing, organizacionales, sociales, entre otras- y niveles -radical o incremental- así como en las posibilidades de utilizar los canales de marketing propios y externos para llevar sus innovaciones al mercado (Christensen et al, 2005 y Chesbrough y Crowther, 2006).

Las empresas, hoy en día, no sólo tienen la oportunidad de obtener conocimientoimportantefueradesímismas 
sino que necesitan mantener ventaja competitiva a través de la colaboración con distintas fuentes de conocimiento tales como centros tecnológicos, universidades, clientes, proveedores de insumos y de tecnologías, intermediarios de innovación y competidores, entre otros.

Por lo ya expresado, este artículo analiza el uso que las medianas y grandes empresas hacen de las diferentes fuentes de la denominada innovación abierta para potenciar su capacidad innovadora y el efecto que esas fuentes tienen en la rentabilidad y la participación de sus mercados.

La fuente de información para este artículo estuvo representada por 434 directivos $^{1}$ de 53 medianas y grandes empresas (de un total de 80 que fueron invitadas a participar) de los sectores alimenticio (7), productos químicos (7), calzado (5), muebles (5), software (4), confección de ropa (4), artes gráficas (4), petróleo (3), productos estéticos (3), metalmecánica (3), marroquinería (3), plástico (2), vidrio (2) y construcción (1), localizadas todas en la ciudad de Bogotá, caracterizadas por contar con más de diez años continuos de actividad y que durante los cinco años previos a este estudio fueron identificadas como empresas innovadoras en diferentes áreas (productos, procesos, marketing u organizacional) por la Encuesta de Desarrollo e Innovación Tecnológica en la Industria Manufacturera (liderada por el Departamento Administrativo Nacional de Estadística de Colombia - Dane) o por el
Premio Accenture a la Innovación $2012^{2}$ o por el Premio Innova - Premio Colombiano a la Innovación Empresarial 2011y 2012.

Para la recolección de la información se aplicó una encuesta de percepción conformada por 30 ítems en escala Likert diseñada específicamente para este estudio -con base en la revisión teórica más adelante presentada- y validada previamente a su aplicación definitiva por un jurado de tres expertos en innovación y por una prueba piloto aplicada a empresas con características similares a las del estudio. La encuesta se aplicó durante el período de marzo de 2011 a noviembre de 2012, en las instalaciones de cada empresa participante, de forma personal por los investigadores y por un grupo de 21 estudiantes de último semestre del programa de Administración de Empresas de la Escuela Internacional de Ciencias Económicas y Administrativas y del Instituto de Posgrados - Forum de la Universidad de La Sabana.

El procesamiento de los datos se realizó mediante la estadística descriptiva y se utilizó el modelo de regresión lineal múltiple para el caso de la prueba de las hipótesis que guiaron el estudio.

\section{Innovación abierta: fundamentación teórica}

De acuerdo con Chesbrough (2003), Gassmann y Enkel (2004), Dahlander y Gann (2010), West y Bogers (2011), Baldwin y von Hippel (2012), Vanhaverbeke y Chesbrough (2013),

1 Al menos un directivo por cada área: administrativa, producción, mercadeo, finanzas y de talento humano con el objetivo de contar con una visión integral de la innovación en la empresa.

2 El "Premio Accenture a la Innovación" fue creado en 2012 por la Consultoría Gerencial de Accenture Colombia con el fin de motivar a las compañías a seguir este camino de progreso, reconociendo y premiando los conceptos empresariales más innovadores implementados en el mercado colombiano. www.premio-innovacion.com 
Felin y Zenger (2014) y Salter, Criscuolo y Ter Wal (2014), el modelo de innovación tradicional, basado en los departamentos de $I+D$, soportado en el aprovechamiento de ideas generadas en el interior de la empresa, orientado a mantener la propiedad intelectual y la innovación bajo control, no es válido para los retos que impone el actual ambiente de los negocios. Para los mencionados autores, este modelo de innovación tradicional se basa en disponer de expertos trabajando en la organización en cada uno de sus diferentes ámbitos para que aporten y desarrollen sus ideas novedosas en función de los planes de la empresa.

En este sentido, la capacidad de generar cambios significativos 0 nuevos productos y servicios, realizar actividades de marketing y obtener organizaciones exitosas, en este modelo, está condicionada a la capacidad de generación de ideas triunfantes por parte de esos expertos en sus respectivos campos, situación esta que no es la apropiada para las exigencias del actual orden económico mundial, caracterizado por la cada vez mayor globalización, la competencia, la incertidumbre, el cambio y el acelerado desarrollo de las tecnologías de la información y las comunicaciones (TIC). Por tanto, urge la necesidad de encontrar un modelo que responda a estas exigencias con base en el conocimiento de los cambios sociales, económicos, tecnológicos $\mathrm{y}$, principalmente, en la disponibilidad del conocimiento mundial.

En respuesta a esta situación ha surgido el denominado enfoque de la innovación abierta, que se ha convertido en uno de los temas más relevantes en la gestión de la innovación desde la publicación realizada sobre el tema por Chesbrough (2003). Esto no quiere decir que la innovación abierta haya surgido recientemente; son diferentes las referencias que durante décadas se hacen a la inclusión de clientes, proveedores de tecnología y de centros de desarrollo en el proceso de innovación realizado por distintas empresas y a la búsqueda de oportunidades de comercializar ideas generadas internamente por una empresa con el apoyo de otras empresas (Von Hippel, 1986; Christensen et al, 2005; Von Hippel, 2010; Dahlander y Gann, 2010 y Salter, Criscuolo y Ter Wal, 2014).

Al respecto, Mowery (2009) afirma que la innovación cerrada (entendida como la innovación que resulta de la generación de ideas dentro de la empresa para diseñar, desarrollar y comercializar sus propios bienes o servicios, por sí mismas) pudo haber sido la excepción en la historia porque, en general, la práctica empresarial se ha caracterizado por su relación con su entorno externo para el desarrollo de sus bienes o sus servicios, lo cual permite inferir que las prácticas de innovación abierta han sido de todos los tiempos, siendo en algunos momentos más intensa que en otros.

Los aportes de la innovación abierta, tal como hoy suele conocerse, son los de haber integrado las diversas actividades ya existentes y otras nuevas que realizan y pueden realizar (de afuera hacia adentro y viceversa) las empresas para fortalecer su capacidad innovadora y de definir una diversidad de métodos, técnicas y estrategias para generar y desarrollar de forma más eficaz las innovaciones (Chesbrough, 2003). Esto representa un nuevo marco para comprender de forma integral el contenido, el contexto y el proceso de innovación en las empresas, desde su interacción con los actores del entorno externo, y hacer cambios fundamentales al modelo de negocios de una empresa (Dahlander y Gann, 2010; Van de Vrande et al, 2009; West y Bogers, 2011 y Huizingh, 2011). 
Según Felin y Zenger (2014), la innovación abierta implica una interacción constante de las empresas con una amplia gama de actores externos incluidos usuarios, clientes, proveedores, universidades, centros privados de investigación, competidores y comunidad en general. Para ello, las empresas disponen, en la actualidad, de mecanismos como alianzas, joint ventures, inversión o acceso a capital social de riesgo, licencias, plataformas de código abierto, participación en diversas comunidades de desarrollo, crowdsourcing, personalización en masa y uso de intermediarios de innovación como InnoCentive, NineSigma o yet2.com, entre otras actividades (Zenger et al, 2011; Afuah y Tucci, 2012; Lakhani et al, 2012 y Baldwin y Von Hippel, 2012).

De acuerdo con Vanhaverbeke y Roijakkers (2013), la innovación abierta es entendida en términos de innovación de adentro hacia afuera y de afuera hacia adentro, donde el conocimiento externo se adquiere para fortalecer las competencias internas y para acelerar el proceso de innovación en la empresa mientras que el conocimiento interno, no explotado por la empresa, es aprovechado por la vía del mercado con socios estratégicos a quienes se les comparte ese conocimiento para ser comercializado por éstos.

Así, el proceso de afuera hacia adentro enriquece la base propia de conocimientos de la empresa a través de la integración de proveedores, clientes, outsourcing de conocimiento, entre otros (Laursen y Salter, 2006; Lettl et al, 2006; Piller y Walcher, 2006; Wijen et al, 2011; Adner, 2012 y Vanhaverbeke y Chesbrough, 2013).

A este respecto, el estudio realizado por Enkel y Gassmann (2008) con 144 empresas, en 2008, reveló que las fuentes de conocimiento externo son los clientes $(78 \%)$, los proveedores $(61 \%)$, los competidores (49\%), el público en general $(37 \%)$, las instituciones de investigación $(28 \%)$, los intermediarios comerciales $(24 \%)$, las consultorías $(21 \%)$ y los socios de otras industrias (14\%).

El proceso de adentro hacia afuera se refiere a llevar al mercado las ideas generadas en el interior de la empresa, no desarrolladas por ésta, mediante la venta de la propiedad intelectual $(\mathrm{PI})$ y la transferencia de su tecnología al ambiente exterior. En este sentido, la empresa no se limita a los mercados en los que participa directamente con sus bienes o servicios sino que lo hace en otros segmentos con la venta de licencias, joint ventures, spinoffs, entre otros (Gassmann y Enkel, 2004 y Lichtenthaler y Ernst, 2009). En su estudio, Enkel y Gassmann (2008) encontraron que el $43 \%$ de las empresas manifestaron tener una política de externalización de sus ideas innovadoras mediante concesión de licencias y otros medios de transferencia de su propiedad intelectual.

El proceso integral de afuera hacia adentro y de adentro hacia afuera se refiere, principalmente, a la creación conjunta de innovaciones con socios complementarios a través de alianzas, cooperaciones $\mathrm{y}$ joint ventures, principalmente. Las empresas que establecen este doble proceso de afuera hacia adentro y de adentro hacia afuera, de manera conjunta, desarrollan y comercializan los resultados de la innovación de forma más eficiente que aquellas que sólo lo hacen en una dirección (Von Hippel y Von Krogh, 2006; Hienerth, 2006; Lettl et al, 2006; Franke et al, 2006; Perkmann y Walsh, 2007; Enkel y Gassmann, 2008 y Müller-Seitz, 2012).

De acuerdo con Enkel, Gassmann y Chesbrough (2009), aunque la era de la innovación abierta comenzó en economías desarrolladas, en empresas y organizaciones que tienen las mejores prácticas en innovación y que producen 
cerca de la mitad de sus innovaciones con ideas generadas fuera de sí mismas, todavía se carece de una comprensión clara por parte de las empresas de las fuentes y los mecanismos, del interior y de fuera de ellas, necesarios para potenciar y desarrollar su capacidad innovadora, es decir, de cómo y dónde beneficiarse plenamente de lo que es la innovación abierta.

De acuerdo con Felin y Zenger (2014), en 2005, Procter and Gamble anunció que fue capaz de aumentar el éxito de sus productos en una tasa del $50 \%$ y la eficiencia de sus actividades de I+D en un $60 \%$ al introducir el concepto de innovación abierta a la organización; en esta misma dirección, en 2007, Philips afirmó que tener una bien establecida relación con su entorno le permitió mejorar su capacidad de innovación en un 57\%, mientras que Siemens manifestó contar con un programa formal de innovación abierta corporativa desde el año 2008 que le ha permitido aumentar su capacidad innovadora en un $64 \%$ y aprovechar más intensamente el conocimiento propio que antes del programa no se utilizaba y que en la actualidad le representa a la empresa un $9,7 \%$ de sus ingresos totales.

De otra parte, al mismo tiempo que las empresas que invierten en actividades de innovación abierta logran grandes beneficios, también se enfrentan a riesgos y barreras que les impiden mayor aprovechamiento de sus iniciativas: el estudio realizado en 2008 por Dahlander y Gann (2010) demostró que los principales riesgos de la innovación abierta son la pérdida del conocimiento propio (48\%), el aumento de los costos de coordinación $(43 \%)$, la pérdida de control y mayor complejidad para administrar los procesos $(41 \%)$, la dificultad en la búsqueda del socio o par adecuado (41\%), el desequilibrio entre las actividades de innovación abierta (36\%), la falta de tiempo y recursos financieros para las actividades de innovación abierta (27\%) y otras barreras (21\%).

Los planteamientos antes presentados sobre la innovación abierta sugieren que las ideas y los conocimientos para la innovación también se encuentran fuera de los límites de la empresa y se pueden exportar desde el interior; así, la I+D externa puede crear un valor significativo para la empresa y ésta puede beneficiarse de la investigación externa y el desarrollo tecnológico. Desde esta perspectiva, la innovación abierta representa un cambio significativo en las prácticas de los profesionales de I+D que son, generalmente, recompensados por el diseño y desarrollo de ideas propias en lugar del desarrollo de ideas tanto propias como externas (Salter, Criscuolo y Ter Wal, 2014).

De acuerdo con Velu, Barrett, Kohli y Oliver (2013), los socios de la innovación abierta varían de proveedores y clientes a las comunidades de aficionados o expertos, las universidades, las instituciones de investigación, las empresas asociadas a otras industrias e incluso los competidores.

Por lo antes mencionado, y dado que la innovación en países como Colombia hace parte de la agenda de la política pública como una estrategia de fomento a la competitividad empresarial y nacional, y la evidencia empírica muestra una relación positiva entre la incorporación de la innovación y la capacidad competitiva empresarial y nacional, este estudio se orientó a probar las siguientes dos hipótesis:

$\mathrm{H} 1$ : Los directivos de las medianas y grandes empresas en Bogotá propician el uso de diferentes fuentes (internas y externas) de innovación en las empresas.

$\mathrm{H} 2$ : El uso de cada una de las fuentes 
(internas y externas) para las innovaciones en las empresas tiene efectos positivos en la rentabilidad y la participación en el mercado.

Para probar estas hipótesis se utilizó el modelo de regresión lineal múltiple de la forma:

$\bar{P}=\beta_{0}+\beta_{1} * X_{1}+\beta_{2} * X_{2}+\beta_{3} * X_{3}+\cdots+\beta_{k} * X_{k}$

Donde:

$\hat{Y}:$ Variable dependiente $=$ innovación desarrollada por las empresas

$\beta_{i} * X_{i}$

: Variable independiente (-ésima) Fuentes internas y externas de innovación $\beta 0$ : Término independiente del modelo

Para el ajuste de los modelos propuestos se utilizó la metodología de mínimos cuadrados cuyos coeficientes de cada variable están dados por:

$$
\hat{\beta}=\left(X^{\prime} X\right)^{-1} * X^{\prime} Y
$$

\section{Donde:}

$\hat{\beta}$ : Vector de coeficientes

$X$ : Matriz de observaciones independientes (Las diversas fuentes de innovación).

$Y$ : Vector de las observaciones dependientes (La innovación, el margen de utilidades y la participación en el mercado).

El nivel de confianza para la aprobación o rechazo de la hipótesis fue de $95 \%$ y donde las probabilidades o valores de $p \leq 0.05$ evidencian existencia de relación entre las variables objeto del análisis.

\section{Innovación abierta en empresas de Bogotá, Colombia}

En esta sección se presentan los resultados obtenidos del estudio realizado, se muestra la percepción de los directivos de las empresas participantes buscando dar respuesta a las hipótesis antes planteadas. El análisis se enfoca a evidenciar si los directivos propician el uso de diferentes fuentes (internas y externas) de innovación en sus empresas $y$, en segundo lugar, a determinar si el uso de esas fuentes tiene efectos positivos en la rentabilidad y la participación en sus mercados.

Uso de fuentes (internas y externas) de innovación en las empresas

Los resultados del estudio indican que en el $67,4 \%$ de las empresas se desarrollaron dos o más innovaciones por año (en el 15,1\% de las empresas, las innovaciones fueron nuevas para el mercado nacional y sólo en el 4,3\% fueron innovaciones para el mercado internacional), en el $80,6 \%$ se introdujeron cambios en los métodos o procedimientos para realizar de forma más efectiva el trabajo, en el $82,6 \%$ se introdujeron cambios en las actividades de marketing y en el $27 \%$ hubo cambios en las actividades relacionadas con la gestión de la compañía.

Estos datos muestran que, en general, las innovaciones en las empresas se hacen en el campo del marketing, seguidas de las de productos, de las de procesos y muy poco en lo referente al campo de la propia gestión de las organizaciones, lo cual significa que las empresas tienden a ser administradas de forma altamente tradicional, situación ésta que si bien es destacable por el interés de las empresas de responder a las necesidades del mercado, también es preocupante porque en un ambiente de negocios altamente dinámico, incierto, globalizado y competitivo como el actual, las organizaciones requieren cambios en sus modelos y paradigmas administrativos como lo plantean Chesbrough (2003), West y Bogers (2011), Baldwin y von Hippel (2012), Vanhaverbeke y Chesbrough 
(2013), Felin y Zenger (2014) y Salter, Criscuolo y Ter Wal (2014).

Por otro lado, las innovaciones desarrolladas por las empresas participantes del estudio, han sido básicamente de tipo incremental y muy poco de tipo radical, situación contraria a la que se plantea con el paradigma de la innovación abierta cuyo propósito se centra en potenciar la capacidad competitiva de las empresas con una mayor capacidad de innovación para el mercado global (Vanhaverbeke y Chesbrough, 2013) y Felin y Zenger, 2014).

Así mismo, las innovaciones realizadas en las empresas están, principalmente, fundamentadas en fuentes internas y limitadas en fuentes externas, situación que se ve reflejada en que las innovaciones en el $77,1 \%$ de las empresas se apoyaron en proyectos realizados en los propios departamentos de I+D, el $71,5 \%$ como consecuencia de la transferencia de tecnología, el $69,2 \%$ de iniciativas de las personas de la empresa, el $43,8 \%$ como consecuencia de la participación de las empresas en redes tecnológicas, el 37,9\% con la utilización de capital de riesgo, el $27,6 \%$ de trabajo conjunto con universidades o centros de investigación, el $26,1 \%$ por la participación en comunidades de desarrollo tecnológico (congresos y ferias) y el $13,6 \%$ por recurrir a intermediarios de la innovación.

De otra parte, las empresas aprovechan muy poco los canales externos para que otras empresas desarrollen sus ideas, por esto sólo el $21,9 \%$ de las empresas manifiestan llevar al mercado las ideas generadas en su interior y no desarrolladas por éstas y ello lo hacen, en un $52,3 \%$, mediante la venta de la propiedad intelectual $(\mathrm{PI})$ el $23 \%$ por la venta de licencias, el $15 \%$ por acuerdos de joint ventures, el $7 \%$ por transferencia de su propiedad intelectual y el $2,7 \%$ mediante otras modalidades como el desarrollo de spinoffs (Tabla 1).

\section{Tabla 1}

\section{Empresas que utilizan fuentes internas y externas de innovación}

\begin{tabular}{lc}
\hline Fuentes de innovación & \% de empresas que usan cada fuente \\
\hline Fuentes internas & 77.1 \\
Departamento de I+D & 69.2 \\
Iniciativas de las personas en la empresa & 37.9 \\
Utilización de capital de riesgo & 71.5 \\
Transferencia de tecnología & 52.3 \\
Venta o transferencia de capital intelectual & \\
Fuentes externas & 3.2 \\
Relación constante con los clientes & 13.6 \\
Participación en redes tecnológicas & 27.6 \\
Intermediarios de innovación & 26.1 \\
Alianzas con universidades y centros de investigación & 3.7 \\
Participación en comunidades de desarrollo & 2.1 \\
Adquisición de capital intelectual &
\end{tabular}

Fuente: Elaboración propia 
Esto muestra que, en general, en las empresas se practica el modelo tradicional de innovación basado en el desarrollo de las propias ideas y muy poco en aprovechar las oportunidades que ofrecen los nuevos modelos de negocios flexibles, abiertos y dinámicos que propone el enfoque de innovación abierta con resultados positivos para las empresas (Dahlander y Gann, 2010 y Salter, Criscuolo y Ter Wal, 2014). De esto se deduce que los directivos de las medianas y grandes empresas en Bogotá propician el uso de las fuentes internas pero poco el de las externas para potenciar su capacidad innovadora, lo cual responde a la hipótesis $(\mathrm{H} 1)$ sin que ello implique aceptar o rechazar está hipótesis.

Aeste respecto, de acuerdo con los resultados de la encuesta, las principales dificultades para utilizar fuentes externas y para transferir conocimiento para la innovación por parte de las empresas con socios externos fueron: $63 \%$ por la falta de confianza entre las empresas, $51 \%$ por la pérdida de control del conocimiento, $43 \%$ por la dificultad en la búsqueda de la fuente o del socio adecuado, 35\% por la falta de tiempo y recursos financieros para las actividades de innovación y $27 \%$ por otros riesgos. Estas dificultades parecen ser motivadas por la idea cultural que en el país se tiene de que dar a conocer las ideas relevantes es el medio para que otros se apropien de ellas sin contraprestación alguna, es decir, esto significa concebir la competencia y el mercado como un espacio de rivales y no de oportunidad para el trabajo conjunto en colaboración con agentes externos.

Efectos de las fuentes internas y externas de innovación en la rentabilidad y participación en los mercados de las empresas
En lo referente a la relación entre la innovación y las fuentes internas y externas para el desarrollo y comercialización de ésta, con un nivel de confianza del $95 \%$ y valores de $p \leq 0.05$, los datos muestran que:

1) Los directivos empresariales manifestaron que la innovación tiene relación positiva con los departamentos de $I+D$, con las iniciativas de las personas dentro de la empresa, con la transferencia de tecnología y con la utilización de capital de riesgo (lo cual se ve reflejado en las probabilidades o valores de $p \leq 0.05^{5}$ ), pero no con la participación en comunidades de apoyo al desarrollo sectorial ni con el uso de intermediarios de la innovación, tampoco con la participación en redes tecnológicas ni con alianzas con universidades o centros de investigación ni, tampoco, con la venta y transferencia de capital intelectual de parte de la empresa hacia socios estratégicos o hacia interesados en las ideas generadas en la empresa pero no aprovechadas por ésta -valores de $p \geq 0.05$ - (Tabla 2) columna derecha).

De las fuentes antes mencionadas, las que más impactan en las actividades de la innovación de las empresas, en su orden son: la existencia de los departamentos de I\&D, las iniciativas de las personas de la empresa, la trasferencia de tecnología y las relaciones con los clientes (Tabla 2, columna de coeficientes - mayores valores de $\beta_{\mathrm{i}) \text {. }}$.

Esta situación es una confirmación del uso del modelo tradicional de innovación centrado en los agentes o factores internos de la empresa mediante un considerable esfuerzo para identificar las necesidades de los usuarios y satisfacerlas a través del mercado. De acuerdo con este enfoque, mientras más se restrinja la propiedad intelectual, en la forma de patentes, 
derechos de autor y marcas registradas, mayor será el incentivo de las empresas para innovar, interpretación esta que, como ya se mencionó, contrasta con el enfoque de innovación abierta basado en el aprovechamiento del conocimiento propio y externo para potenciar la capacidad de innovación mediante la creación de valor a través de la interacción entre los distintos agentes de los entornos interno y externo.

\section{Tabla 2}

\section{Relación entre innovación y fuentes internas y externas}

\begin{tabular}{|c|c|c|c|}
\hline Fuentes de innovación & Coeficientes $/ \boldsymbol{\beta}_{i}$ & Estadístico $t$ & Probabilidad/P \\
\hline Intercepción & 1.20027547 & 4.21573985 & 3.3633E-05 \\
\hline \multicolumn{4}{|l|}{ Fuentes internas } \\
\hline Departamento de I+D & 0.27036037 & 4.63973572 & 5.3633E-06 \\
\hline Iniciativas de las personas en la empresa & 0.1729128 & 3.6425026 & 0.00032163 \\
\hline Utilización de capital de riesgo & 0.11510825 & 2.02723257 & 0.04358584 \\
\hline Transferencia de tecnología & 0.16951278 & 2.73714109 & 0.00659421 \\
\hline Venta o transferencia de capital intelectual & 0.08365376 & 1.46284807 & 0.14463052 \\
\hline \multicolumn{4}{|l|}{ Fuentes externas } \\
\hline Relación constante con los clientes & 0.12960735 & 2.69979482 & 0.00736047 \\
\hline Participación en redes tecnológicas & -0.08365376 & -1.46284807 & 0.14463052 \\
\hline Intermediarios de innovación & 0.08532153 & 1.34549103 & 0.17955528 \\
\hline $\begin{array}{l}\text { Alianzas con universidades y centros de } \\
\text { investigación }\end{array}$ & 0.05475083 & 0.93917522 & 0.34845025 \\
\hline Participación en comunidades de desarrollo & 0.09621358 & 1.30224555 & 0.19390263 \\
\hline
\end{tabular}

Fuente: Elaboración propia

En general, los datos de la Tabla 2 muestran que, respecto de la segunda hipótesis $(\mathrm{H} 2)$, con un nivel de confianza del $95 \%$ y valores de $p \leq 0.05$ existe relación positiva entre el uso de las fuentes internas de innovación y la innovación empresarial pero no entre el uso de las fuentes externas y la innovación (valores de $P \geq 0.05$ ).

2) El margen de utilidades de las empresas está relacionado con las actividades de los departamentos de I+D, la transferencia de tecnología, la iniciativa de las personas de la empresa y las relaciones constantes con los clientes (valores de $p \leq 0.05$ ), pero no con actividades tales como la utilización de capital de riesgo, la participación en redes tecnológicas, el uso de intermediarios de innovación, las alianzas con universidades o centros de investigación, la participación en comunidades de desarrollo y la venta o transferencia de capital intelectual de unas a otras empresas - valores de $p \geq 0.05$ (Tabla 3, Columna de probabilidades).

De esas fuentes de innovación, las que tienen impacto positivo en los márgenes de utilidad de las empresas son, 
las relaciones frecuentes con los clientes y las actividades de los departamentos de $I \& D$, mientras que, las de impacto negativo son, la utilización de capital de riesgo y la participación en redes tecnológicas (Tabla 3 , columna de coeficientes - valores de $\beta \mathrm{i}$ ).

Esto corrobora que las empresas para generar, diseñar y desarrollar innovaciones, en general, recurren a las fuentes tradicionales como son los departamentos de $1+D$, las ideas de sus propios trabajadores y los contactos con los clientes, pero desaprovechan toda una variedad de fuentes externas (como los intermediarios de innovación) que son hoy en día cada vez más utilizadas por las empresas en los países desarrollados para obtener de estas fuentes más potencialidad de innovación, con menor costo y menor riesgo, en tiempos más cortos (Baldwin y Von Hippel, 2012;
Vanhaverbeke y Chesbrough, 2013; Felin y Zenger, 2014 y Criscuolo y Ter Wal, 2014).

Esta tendencia a utilizar exclusivamente las fuentes habituales para la innovación puede obedecer al estilo tradicional de administrar las organizaciones por parte de sus directivos, al desconocimiento de la existencia de las diversas alternativas que hoy pueden utilizar las empresas para potenciar su capacidad competitiva y a la falta de experiencia de las empresas para trabajar de forma conjunta con otras organizaciones (empresas, instituciones académicas, intermediarios de innovación, entre otras) con el objetivo de desarrollar innovaciones y aprovechar los canales de otras empresas para difundir las ideas generadas en su interior.

\section{Tabla 3}

\section{Relación entre margen de utilidad y fuentes de innovación}

\begin{tabular}{|c|c|c|c|}
\hline Fuentes de innovación & Coeficientes $/ \boldsymbol{\beta}_{i}$ & Estadístico $t$ & Probabilidad/P \\
\hline Intercepción & 1.89780181 & 10.9348595 & 2.0671E-23 \\
\hline \multicolumn{4}{|l|}{ Fuentes internas } \\
\hline Departamentos de I+D & 0.1094837 & 2.4309468 & 0.0156873 \\
\hline Transferencia de tecnología & 0.0762575 & 2.0855477 & 0.0379250 \\
\hline Iniciativas de las personas en la empresa & 0.0574592 & 1.9856458 & 0.0480479 \\
\hline Utilización de capital de riesgo & -0.0292284 & -0.7742282 & 0.4394491 \\
\hline Venta o transferencia de capital intelectual & 0.0500157 & 0.8600900 & 0.3904747 \\
\hline \multicolumn{4}{|l|}{ Fuentes externas } \\
\hline Participación en redes tecnológicas & -0.0087105 & -0.2365348 & 0.81319069 \\
\hline Relación constante con los clientes & 0.12446811 & 2.07311291 & 0.03907631 \\
\hline Intermediarios de innovación & 0.05013452 & 1.29696589 & 0.19571063 \\
\hline $\begin{array}{l}\text { Alianzas con universidades y centros de } \\
\text { investigación }\end{array}$ & 0.0241436 & 0.67940201 & 0.49744441 \\
\hline Participación en comunidades de desarrollo & 0.04310274 & 0.93658641 & 0.34977844 \\
\hline
\end{tabular}

Fuente: Elaboración propia 
En síntesis, los datos de la Tabla 3 muestran que, respecto de la segunda hipótesis $(\mathrm{H} 2)$, con un nivel de confianza del $95 \%$ y valores de $p \leq 0.05$ las fuentes internas de innovación, los departamentos de $I+D$, las iniciativas de las personas de la empresa y la transferencia de tecnologías tienen relación directa con el margen de utilidad de las empresas, pero, de las fuentes externas, sólo la relación constante con los clientes tiene efectos positivos con dicho margen.

3) Situación similar se da para la relación entre las fuentes de innovación y la participación en el mercado en donde la percepción de los directivos evidencia que de la diversidad de fuentes internas y externas que en la actualidad disponen las empresas para potenciar su capacidad innovadora, sólo los departamentos de $I+D$, las iniciativas de las personas de la empresa y la constante interacción con los clientes tienen relación con la capacidad de la empresa para ganar participación en el mercado (Tabla 4), situación ésta que difiere de los resultados de los estudios de Velu, Barrett, Kohli y Oliver (2013) sobre el tema dado que en éstos se muestra que las empresas que utilizan las fuentes tradicionales y las no tradicionales, para potenciar su capacidad de innovación, tienden a incrementar su participación en el mercado.

De las fuentes de innovación que tienen relación con la participación en el mercado de las empresas, sólo se destacan las que tienen que ver con las relaciones frecuentes con los clientes, las iniciativas de las personas de la empresa y las actividades de los departamentos de I\&D. (Tabla 3, columna de coeficientes valores de $\beta$ ).

\section{Tabla 4}

\section{Relación entre participación en el mercado y fuentes de innovación}

\begin{tabular}{|c|c|c|c|}
\hline Fuentes de innovación & Coeficientes $/ \boldsymbol{\beta}_{i}$ & Estadístico $t$ & Probabilidad/P \\
\hline Intercepción & 1.73258344 & 11.180645 & 3.0708E-24 \\
\hline \multicolumn{4}{|l|}{ Fuentes internas } \\
\hline Departamentos de I+D & 0.07160898 & 2.25786269 & 0.02472434 \\
\hline Transferencia de tecnología & 0.02122127 & 0.62957287 & 0.52948744 \\
\hline Iniciativas de las personas en la empresa & 0.08097196 & 2.01358987 & 0.04500919 \\
\hline Utilización de capital de riesgo & 0.02155369 & 0.66019014 & 0.50967454 \\
\hline Venta o transferencia de capital intelectual & 0.03803181 & 0.79786851 & 0.42562293 \\
\hline \multicolumn{4}{|l|}{ Fuentes externas } \\
\hline Participación en redes tecnológicas & 0.06388144 & 1.94284781 & 0.0530368 \\
\hline Relación constante con los clientes & 0.10495712 & 2.08281599 & 0.03817542 \\
\hline Intermediarios de innovación & -0.00935973 & -0.27118451 & 0.78644897 \\
\hline $\begin{array}{l}\text { Alianzas con universidades y centros de investi- } \\
\text { gación }\end{array}$ & 0.03533737 & 1.11370439 & 0.26636115 \\
\hline Participación en comunidades de desarrollo & 0.09027374 & 1.94952375 & 0.05223107 \\
\hline
\end{tabular}

Fuente: Elaboración propia. 
Finalmente, los datos de la Tabla 4 indican que, respecto de la segunda hipótesis ( $\mathrm{H} 2)$, con un nivel de confianza del $95 \%$ y valores de $p \leq 0.05$, de las fuentes internas de innovación sólo los departamentos de $\mathrm{I}+\mathrm{D}$ tienen relación directa con la participación en el mercado de las empresas $y$, de las fuentes externas, sólo la relación constante con los clientes tiene efectos positivos en esa participación.

\section{Conclusiones}

En las empresas estudiadas es poco practicado el uso de fuentes externas de innovación que les permita aprovechar las oportunidades para su propia innovación. Esta situación contrasta con los resultados de los estudios realizados sobre los usos de las fuentes internas y externas de innovación por parte de empresas de los países desarrollados para potenciar su capacidad de innovación dada la tendencia al uso de distintas nuevas fuentes, no tanto de las tradicionales.

Por esto, llama la atención la manera tradicional como se sigue desarrollando la innovación en las empresas estudiadas por cuanto se evidencia que requieren un sistema de innovación más ágil, flexible y menos costoso para responder de forma competitiva a las actuales y futuras exigencias del nuevo ambiente de los negocios. Hoy se reconoce que para asegurarse de que se mantienen en la vanguardia de su sector, las compañías cada vez deben ser más flexibles y abiertas al acervo del talento disponible en el entorno (regional, nacional y global).

De ello surge la necesidad de que las empresas de las economías emergentes analicen la pertinencia $y$ conveniencia de incorporar el enfoque de innovación abierta entre sus estrategias orientadas a mejorar su capacidad competitiva pero cuidando que la novedad del tema no se convierta en una moda más en la gerencia de estas empresas, como suele suceder con los diversos enfoques gerenciales que se transfieren de las economías desarrolladas, de forma acrítica, sin evaluar la pertinencia y la adaptación a las necesidades y particularidades de las empresas a las que se aplica, dejando en claro que este enfoque es otra estrategia con la que hoy cuentan las empresas para potenciar su capacidad competitiva pero no la panacea para resolver las diferentes dificultades a las que éstas se deben enfrentar.

Es necesario que estas empresas comiencen a tener conciencia de la importancia de trabajar de forma colaborativa con los diferentes agentes de su entorno externo (local, regional, nacional y global) con el fin de disponer de un mejor conocimiento para la toma de decisiones y de conseguir de forma eficaz los objetivos corporativos logrando así mejorar su capacidad competitiva. Las empresas requieren comenzar a reconocer que la colaboración está convirtiéndose en una importante fuente de ventaja competitiva que contrasta con la forma individualista tradicional de trabajar y producir, no viable para enfrentar los retos del actual y futuro orden económico caracterizado por la cada vez mayor globalización, mayor competencia, mayor incertidumbre y unos rápidos y complejos cambios promovidos y facilitados por los acelerados desarrollos de las TIC.

Las empresas, en países como Colombia, requieren entender que la estrecha colaboración con los diferentes agentes del entorno externo es una eficaz estrategia para captar ideas y expandir sus mercados actuales o 
acceder a otros nuevos y que, mediante la colaboración, sus innovaciones pueden llegar al mercado a través de diversos canales, bien sea a través de una spinoff, una licencia, una venta final, una donación o como input para otras innovaciones.

En otras palabras, las empresas tienen que aprender que el trabajo colaborativo no significa restarle importancia a los departamentos de I+D internos sino que es saber aprovechar las oportunidades que ofrece este enfoque para multiplicar las potencialidades de estos departamentos explotando colaboraciones externas en las diferentes fases del proceso de innovación, desde la investigación hasta el desarrollo de los productos y su posterior comercialización.

En este nuevo entorno, las empresas necesitan conocer y aprovechar adecuadamente las oportunidades existentes en sus redes de innovación de tal modo que es importante que las empresas analicen la conveniencia de pasar de un modelo de innovación cerrado a uno abierto que les demanda cambios no sólo en el propio proceso de innovación sino en el de la administración. Por consiguiente, los cambios deben ser estructurales, desde la cultura organizacional hasta el modelo de negocio, pasando por la tecnología, la gestión de la propiedad intelectual, principalmente; se trata, por tanto, de propiciar un cambio tal del paradigma imperante en las empresas que les permita ser más eficaces y más competitivas mediante la generación sostenible de valor soportada en la innovación para cada uno de los diferentes stakeholders.

En síntesis, desde esta concepción de la innovación, la sociedad, en su conjunto, ha de estar abierta a los nuevos conocimientos, a las reglas y a los valores de la investigación y a la innovación con el convencimiento de que todas las instituciones y organizaciones, en acción conjunta, pueden ser más innovadoras en los diferentes ámbitos (sociales, culturales, económicos, políticos, entre otros) y más competitivas de forma sostenible e integral.

También es importante señalar que el estudio se centró en el análisis de los resultados de la percepción de los directivos de una muestra de medianas y grandes empresas de distintos sectores económicos y diferente naturaleza jurídica, lo que limita su generalización dado que los resultados pueden ser distintos si se analizan las empresas por sectores de actividad, por tamaño y si se analizan, en particular, las variables estudiadas mediante estudios de caso en profundidad.

También, el estudio se limitó a empresas de una determinada región del país, situación que dificulta las generalizaciones de las conclusiones a otros contextos, por lo cual otra futura investigación puede ser la de realizar estudios que incluyan empresas de distintas regiones del país o empresas de países similares a Colombia y que permitan hacer análisis comparativos.

No puede dejarse de lado que otras variables importantes por estudiar en este tema son el impacto de la cultura organizacional y la de la propia sociedad en la adopción de la innovación abierta como una estrategia de innovación para las empresas de la región y las estrategias empresariales y del Estado que pueden incentivar el uso de este tipo innovación en las empresas en los países emergentes. 


\section{Referencias bibliográficas}

Adner, Ron (2012), The wide lens: A new strategy for innovation. New York: Portfolio/Penguin.

Afuah, Allan y Tucci, Cristopher (2012), Crowdsourcing as a solution to distant search. Academy of Management Review. Vol. 37. Academy of Management. (355-375).

Baldwin, Carliss, y von Hippel, Eric (2012), Modeling a paradigm shift: from producer innovation to user and open collaboration. Journal Organization Science. Vol. 22, No 6. Institute for Operations Research and the Management Sciences. (1399-1417).

Chesbrough, Henry (2003), Open Innovation: The New Imperative for Creating and Profiting from Technology. Boston: Harvard Business School Press.

Christensen, Jens, Olesen Michael y Kjær Jonas (2005), The industrial dynamics of open innovation - evidence from the transformation of consumer electronics. Research Policy, Vol. 34, No 10. Elsevier- ScienceDirect. (1533-1549).

Dahlander, Linus y Gann, David (2010), How open is innovation? Research Policy, Vol. 39. Elsevier- ScienceDirect. (699-709).

Enkel, Ellen Gassmann, Oliver y Chesbrough, Henry (2009), Open R\&D and open innovation: exploring the phenomenon. R\&D Management, Vol 39, No 4, John Wiley \& Sons Ltd y RADMA. (311- 316).

Enkel, Ellen y Gassmann, Oliver (2008), Driving open innovation in the front end. The IBM case. Working Paper University of St. Gallen and Zeppelin University, St. Gallen and Friedrichshafen. Paris, France: EURAM.

Felin, Teppo y Zenger, Todd (2014), Closed or open innovation? Problem solving and the governance choice. Research Policy, Vol. 43, No 21, ElsevierScienceDirect. (914-925).

Franke, Nikolaus, von Hippel, Erik y Schreier, Martin (2006), Finding commercially attractive user innovations: a test of lead-user theory. Journal of Product Innovation Management, Vol. 23, No 4, John Wiley \& Sons Ltd and RADMA. (301-315)

Gassmann, Oliver y Enkel, Ellen (2004), Towards a theory of open innovation: three core process archetypes. Proceedings of the R\&D Management Conference, Lisbon, Portugal, July 6-9. Paris, France: University of St. Gallen

Hienerth, Christoph (2006), The commercialization of user innovations: the development of the rodeo kayak industry. R\&D Management, Vol. 36, No 3, John Wiley \& Sons Ltd and RADMA. (273-294).

Huizingh, Eelko (2011), Open innovation: State of the art and future perspectives. Technovation. Vol. 31. Elsevier Ltd. (2-9.

Lakhani, Karim, Hifshitz-Assaz, y Tushman, Michael (2012), Open Innovation and Organizational Boundaries: The Impact of Task Decomposition and Knowledge Distribution on the Locus of Innovation. Harvard Business School, Working paper.

Laursen, Keld y Salter, Ammon (2006), Open for innovation: the role of openness in explaining innovation performance among UK manufacturing firms. Strategic Management Journal, Vol. 27, No 2, John Wiley \& Sons Ltd. (131-150).

Lettl, Cristopher, Herstatt, Comelius y Gemuenden, Hans (2006), Users' contributions to radical innovation: evidence from four cases in the field of medical equipment technology. R\&D Management, Vol.36, No 3, John Wiley \& Sons Ltd. (251-272).

Lichtenthaler, Ulrich y Ernst, Holder (2009), Opening up the innovation process: the role of technology aggressiveness. R\&D Management. Vol. 39, No1, John Wiley \& Sons Ltd. (38-54).

Mowery, David (2009), Plus and change: industrial $R \& D$ in the third industrial revolution. Industrial and Corporate Change. Vol.18, No 1. Oxford Journals. (1-50). 
Müller-Seitz, Gordon (2012), Absorptive and desorptive capacity - related practices at the network level - the case of SEMATECH. R\&D Management. Vol. 42, No 1. John Wiley \& Sons Ltd y RADMA. (90-99).

Perkmann, Markus y Walsh, Kathrin (2007), University-industry relationships and open innovation: towards a research agenda. International Journal of Management Reviews, Vol.9, No 4, John Wiley \& Sons Ltd. (259-280).

Piller, Frank y Walcher, Dianne (2006), Toolkits for idea competitions: a novel method to integrate users in new product development. R\&D Management, Vol. 36, No 3, John Wiley \& Sons Ltd and RADMA. (307-318).

Salter, Ammon; Criscuolo, Paola y Ter Wal, Anne (2014), Coping with Open Innovation: Responding to the Challenges of External Engagement in R\&D. California Management Review, Vol. 56, No 2. University of California. (77-94).

Van de Vrande, Vadeska; de Jong, Jeroen; Vanhaverbeke, Wim y de Rochemont, Maurice (2009), Open innovation in SMEs: trends, motives and management challenges. Technovation, Vol. 29, Elsevier Ltd. (423-437).

Vanhaverbeke, Wim y Roijakkers, Nadine (2013), Enriching Open Innovation Theory and Practice by Strengthening the Relationship with Strategic Thinking. En: www. springer783642414787-c2.pdf? Consultado el 9 de agosto de 2014.

Vanhaverbeke, Wim y Chesbrough, Henry (2013), A classification of open innovation and open business models. In Chesbrough, Henry and Vanhaverbeke, Wim \& J. West (Eds.), Exploring the next wave of open innovation research. Oxford: Oxford University Press.

Vanhaverbecke, Wim, Van de Vrade, Valeska y Chesbrough, Henry (2008),
Understanding the advantages of open innovation practices in corporate venturing in terms of real options. Creativity and Innovation Management, Vol. 17, No 4, John Wiley \& Sons Ltd. (251-258).

Velu, Chander; Barrett, Michael; Kohli, Rajiv y Oliver, Torsten (2013), Thriving in Open Innovation Ecosystems: Toward a Collaborative Market Orientation. Cambridge: University of Cambridge.

Von Hippel, Eric (1986), Lead users: a source of novel product concepts. Management Science. Vol. 32, No 7, Management Science. (791-805).

Von Hippel, Eric y von Krogh, George (2006), Free revealing and the privatecollective model for innovation incentives. R\&D Management, Vol.36, No 3, John Wiley \& Sons Ltd. (295-306).

Von Hippel, Eric (2010), Comment on 'Is open innovation a field of study or a gggcommunication barrier to theory developent?' Technovation. Vol. 30, Elsevier Scientific Publishing. (555).

West, Joel y Bogers, Marcel (2011), Profiting from external innovation: a review of research on open innovation. In: 9th International Open and User Innovation Workshop, Vienna, Austria, En: http://ssrn.com/ abstract $=1949520$. Consultado el 19 de agosto de 2013.

Wijen, Frank, Noorderhaven, Niels, y Vanhaverbeke, Wim (2011), Structural antecedents of corporate network evolution. International Journal of Business Environment. Vol. 4, No 3. Inderscience Publishers, (207-233).

Zenger, Todd; Felin, Teppo, y Bigelow, Lyda (2011), Theories of the firm-market boundary. Academy of Management Annals. Vol. 5, No 1. Washington University (89-113). 